\title{
Perfil clínico e epidemiológico dos casos de hanseníase em pacientes adultos em um município do Maranhão
}

\author{
Clinical and epidemiological profile of leprosy cases in adult patients in a municipality in \\ Maranhão
}
Perfil clínico y epidemiológico de los casos de lepra en pacientes adultos en un municipio de Maranhão

Letícia Samara Ribeiro da Silva ${ }^{1 *}$, Claudiane Sousa Miritiba ${ }^{1}$, Karla Janyelle de Sousa da Silva ${ }^{1}$, Iracema Murada Pessoa ${ }^{1}$, Andressa Arraes Silva', Mara Julyete Arraes Jardim², Luciane Sousa Pessoa Cardoso ${ }^{1}$, Naylanny Gonçalves Torres Cunha ${ }^{1}$, Larissa Silva Oliveira ${ }^{1}$, Railda Lima Rodrigues'.

\section{RESUMO}

Objetivo: Analisar o perfil clínico e epidemiológico dos casos de Hanseníase em pacientes adultos em um município do Maranhão. Métodos: Trata-se de um estudo retrospectivo, de caráter descritivo, com abordagem quantitativa e de cunho epidemiológico, realizado com base em dados obtidos através do Sistema de Informação de Agravos de Notificação (SINAN) referente aos casos de Hanseníase em adultos no município maranhense, entre 2016 e 2018. Por constituir uma pesquisa a partir de dados secundários e de domínio público, não houve a necessidade de apreciação e aprovação pelo Comitê de Ética em Pesquisa. Resultados: Foram registrados 145 casos de Hanseníase em adultos, com predominância do sexo masculino (68\%), na faixa etária de 30 a 39 anos (29\%), com classificação operacional multibacilar (85\%) e forma clínica dimorfa (67\%), modo de entrada como caso novo (72\%), cuja detecção acontece em sua maioria por demanda espontânea (41\%), com grau de incapacidade zero (41\%) e tipo de saída por cura (62\%). Conclusão: Esperase que este estudo forneça informações que permitam conhecer a dimensão dessa doença na coletividade, a fim de potencializar medidas de prevenção e o pronto tratamento dos casos iniciais, o exame periódico dos contatos e a educação em saúde para a população.

Palavras-chave: Saúde do adulto, Hanseníase, Enfermagem em saúde comunitária.

\begin{abstract}
Objective: To analyze the clinical and epidemiological profile of leprosy cases in adult patients in a city in Maranhão. Methods: This is a retrospective, descriptive study, with a quantitative and epidemiological approach, carried out based on data obtained through the Information System for Notifiable Diseases (SINAN) referring to Hansen's disease cases in adults in the municipality of Maranhão, among 2016 and 2018. As it constitutes a research based on secondary and public domain data, there was no need for appreciation and approval by the Research Ethics Committee. Results: 145 leprosy cases were recorded in adults, with a predominance of males (68\%), aged 30 to 39 years (29\%), with multibacillary operational classification $(85 \%)$ and a dimorphic clinical form (67\%), entry mode as a new case $(72 \%)$, the detection of which happens mostly by spontaneous demand (41\%), with zero degree of disability ( $41 \%)$ and type of exit due to cure (62\%). Conclusion: It is hoped that this study will provide information that will allow to know the dimension of this disease in the community, in order to enhance preventive measures and the prompt treatment of initial cases, the periodic examination of contacts and health education for the population.
\end{abstract}

Keywords: Adult health, Hansen's disease, Community health nursing.

1 Universidade Estadual do Maranhão (UEMA), Bacabal - MA. *E-mail: leticiasamaras2@outlook.com

2 Universidade Estadual do Maranhão (UEMA), Coroatá - MA. 


\section{RESUMEN}

Objetivo: Analizar el perfil clínico y epidemiológico de los casos de lepra en pacientes adultos en una ciudad de Maranhão. Métodos: Este es un estudio descriptivo retrospectivo, con un enfoque cuantitativo y epidemiológico, realizado con base en datos obtenidos a través del Sistema de Información para Enfermedades de Notificación (SINAN) que se refieren a casos de enfermedad de Hansen en adultos en el municipio de Maranhão, entre 2016 y 2018. Como constituye una investigación basada en datos de dominio público y secundario, no hubo necesidad de apreciación y aprobación por parte del Comité de Ética en Investigación. Resultados: Se registraron 145 casos de lepra en adultos, con predominio de varones (68\%), de 30 a 39 años (29\%), con clasificación operativa multibacilar (85\%) y una forma clínica dimórfica (67\%), modo de entrada como un nuevo caso $(72 \%)$, cuya detección ocurre principalmente por demanda espontánea (41\%), con cero grado de discapacidad (41\%) y tipo de salida debido a la cura (62\%). Conclusión: Se espera que este estudio proporcione información que permita conocer la dimensión de esta enfermedad en la comunidad, a fin de mejorar las medidas preventivas y el tratamiento oportuno de los casos iniciales, el examen periódico de contactos y la educación sanitaria para la población.

Palabras clave: Salud de adultos, Enfermedad de Hansen, Enfermería de salud comunitaria.

\section{INTRODUÇÃO}

A Hanseníase, conhecida anteriormente como Lepra, é considerada uma das enfermidades mais antigas da história da humanidade. É uma doença crônica e infectocontagiosa causada pelo Mycobacterium leprae ou bacilo de Hansen, uma bactéria intracelular obrigatória, que se manifesta principalmente através de sinais e sintomas dermatoneurológicos, com predileção por células cutâneas e dos nervos periféricos. Com um período de incubação que varia de 2 a 7 anos, é uma doença de evolução lenta e silenciosa que pode acometer pessoas de ambos os sexos e em qualquer faixa etária, sendo mais incidente em adultos (BRASIL, 2017; BASSO MEM e SILVA RLF, 2017).

De acordo com o Ministério da Saúde (BRASIL, 2017), a Hanseníase é adquirida por meio do contato próximo e prolongado de uma pessoa suscetível com um doente que não está fazendo o tratamento. A pessoa com a enfermidade elimina o bacilo pelas vias respiratórias superiores (mucosa nasal e orofaringe) por meio da tosse ou espirro. Quanto ao diagnóstico, tem como base aspectos clínicos (sinais e sintomas) evidenciados por lesões de pele ou áreas com sensibilidade alterada e/ou comprometimento dos nervos periféricos, além de aspectos epidemiológicos que envolvem o conhecimento da história e situação de vida da pessoa acometida pela doença (VELÔSO DS, et al., 2018; QUARESMA MSM, et al., 2019).

Com relação à sua epidemiologia, no Brasil, a Hanseníase constitui um expressivo problema de saúde pública devido a sua magnitude e alto poder incapacitante. Somado a isso, continua sendo uma doença bastante prevalente apesar dos programas, ações de controle e das metas de eliminação dessa patologia no país (BRASIL, 2018). Por ser uma doença de notificação compulsória e de investigação obrigatória em todo o território nacional, os casos diagnosticados de Hanseníase devem ser notificados utilizando-se a ficha de Notificação/Investigação do Sistema de Informação de Agravos de Notificação - SINAN (BRASIL, 2016).

Segundo a Organização Mundial da Saúde (OMS), em 2016, 143 países reportaram 214.783 casos novos de Hanseníase, representando uma taxa de detecção de 2,9 casos por 100 mil habitantes. No mesmo ano, foram notificados 25.218 casos novos da doença no Brasil, atingindo uma taxa de detecção de 12,2 casos por 100 mil habitantes (CUNHA DV, et al., 2019). O Brasil mantém, nas últimas décadas, a situação mais desfavorável na América e o diagnóstico de segunda maior quantidade de casos novos no mundo, perdendo apenas para a Índia (PORTO MJ, et al., 2015; LAFAIETE C, 2019). Diante dessas informações, é comprovado que esses parâmetros classificam o país como de alta carga para a doença.

No cenário brasileiro, as regiões Sudeste e Sul já obtiveram êxito na redução acentuada de casos da Hanseníase, inclusive com coeficientes de prevalência abaixo da média nacional. No entanto, as regiões Norte, Centro-Oeste e Nordeste ainda apresentam altos patamares de casos da doença. Apesar da tendência de estabilização dos coeficientes de detecção no Brasil, essa variação nos índices evidencia a relação das 
desigualdades regionais de desenvolvimento social e econômico com a história epidemiológica das doenças infectocontagiosas presentes no país (RIBEIRO MDA, et al., 2018; SOUZA EA, et al., 2018). Assim, a dimensão sociocultural, ambiental e o padrão epidemiológico desigual da doença ainda constituem um desafio para os profissionais de saúde e para os próprios pacientes (PASSOS CEC, et al., 2016).

No período de 2012 a 2016, o Nordeste encontrava-se em terceiro lugar com relação à taxa média de detecção de casos novos de Hanseníase, correspondendo a 23,42 casos novos a cada 100 mil habitantes, o que justifica a classificação "muito alta" no período. Fortalecendo tal estatística, no mesmo período, o estado do Maranhão encontrava-se como $2^{\circ}$ colocado do Brasil em detecção de casos novos e, no geral, o mais prevalente do Nordeste.

O Maranhão apresenta tendência decrescente de seus indicadores de detecção de casos novos, entretanto, no período de 2012 a 2016, esse coeficiente apresentou o valor de 53,91 casos/100 mil habitantes, apresentando classificação "hiperendêmica" (>40,0/100 mil hab) de acordo com os parâmetros oficiais do Ministério da Saúde (BRASIL, 2018). Como afirmam Rosa AM, et al. (2019), segundo registros do Maranhão, das 19 Regiões de Saúde do estado, 08 apresentam situação hiperendêmica, dentre elas a do município de Bacabal.

Diante desse cenário, considerando a importância de estudos epidemiológicos para a obtenção de dados sobre o perfil dos portadores da doença e sua tendência de acometimento, além de auxiliar na compreensão dos fatores que contribuem para a manutenção da endemicidade e subsidiar a implementação de ações para sua prevenção e controle, este artigo tem como objetivo analisar o perfil clínico e epidemiológico dos casos de Hanseníase em pacientes adultos em um município do Maranhão, no período de 2016 a 2018.

\section{MÉTODOS}

Trata-se de um estudo retrospectivo, de caráter descritivo, com abordagem quantitativa e de cunho epidemiológico realizado com base em dados de casos notificados de Hanseníase em pacientes adultos em um município do Maranhão, entre 2016 e 2018. Os dados foram coletados no mês de junho de 2019 e obtidos eletronicamente a partir do Sistema de Informação de Agravos de Notificação (SINAN), por meio do acesso ao Departamento de Informática do Sistema Único de Saúde (DATASUS). Foram incluídos todos os casos de Hanseníase em adultos notificados no município no período estabelecido. Foram excluídos todos os casos notificados que não fossem da idade adulta, bem como as notificações que estiveram fora do período do estudo.

Levando em consideração a dimensão da Hanseníase como uma das doenças definidas como prioritárias, mas inserida como negligenciada, o presente estudo tem como pergunta norteadora: Qual o perfil clínico e epidemiológico dos casos de Hanseníase em pacientes adultos em um município do Maranhão, no período de 2016 a 2018 ?

Realizou-se a coleta dos dados para se obter as informações sobre a temática em estudo, mediante o acesso ao item "Informações de Saúde (TABNET)" em "Epidemiológicas e Morbidade" e a seleção do tópico "Casos de Hanseníase (SINAN)". Para atender ao objetivo do estudo, foram selecionadas as seguintes variáveis: sexo (feminino e masculino); faixa etária (20 a 29 anos, 30 a 39 anos, 40 a 49 anos e 50 a 59 anos); classificação operacional (Paucibacilar e Multibacilar); formas clínicas (Indeterminada, Tuberculoide, Dimorfa e Virchowiana); modo de entrada (Caso Novo, Transferência do mesmo município, Transferência de outro município - mesma Unidade da Federação (UF), Transferência de outro estado, Recidiva); modo de detecção (Encaminhamento, Demanda espontânea, Exame de contatos); grau de incapacidade física (GIF) (Grau 0, Grau 1, Grau 2); e tipo de saída (Cura, Transferência para mesmo município, Transferência para outro município, Óbito, Abandono).

Os dados foram tabulados e analisados utilizando-se o software Microsoft Excel, versão 2010, favorecendo a análise exploratória e estatística das variáveis apresentadas em frequência absoluta e relativa percentual com auxílio de tabelas. Como este estudo se baseou em dados disponibilizados pelo Ministério da Saúde em meio eletrônico, os quais são de domínio público, além de ser mantido o sigilo sobre as informações de identificação dos pacientes, não houve a necessidade de apreciação e aprovação pelo Comitê de Ética em Pesquisa. 


\section{RESULTADOS E DISCUSSÃO}

No período de 2016 a 2018, foram notificados 225 casos de Hanseníase no município maranhense. Desse total, 145 casos eram de pacientes adultos, correspondendo a $64 \%$. Observou-se uma diminuição no número de casos da doença no decorrer dos anos, passando de 81 casos no ano de 2016 para 65 em 2018. No entanto, quando se considera o número de casos em adultos, os valores permaneceram próximos (Tabela 1).

Tabela 1 - Número de casos de Hanseníase notificados segundo ano de ocorrência. 2016-2018.

\begin{tabular}{ccc}
\hline Ano & Número de casos & Número de casos em adultos \\
\hline 2016 & 81 & 51 \\
2017 & 79 & 47 \\
2018 & 65 & 47 \\
\hline Total & 225 & 145
\end{tabular}

Fonte: Silva LSR, et al., 2020. SINAN, 2019.

Quando se analisou a proporção de casos de Hanseníase segundo gênero, verificou-se predominância do sexo masculino com 99 casos (68\%). Em relação à idade, houve maior prevalência na faixa etária de 30 a 39 anos, com 42 casos (29\%) (Tabela 2).

Tabela 2 - Perfil dos pacientes adultos portadores de Hanseníase segundo sexo e faixa etária. 2016-2018.

\begin{tabular}{ccc}
\hline Variável & $\mathbf{n}$ & $\%$ \\
\hline Sexo & & 68 \\
Masculino & 99 & 32 \\
\hline Feminino & 46 & 23 \\
\hline Faixa etária & & 29 \\
\hline 20 a 29 anos & 34 & 27 \\
30 a 39 anos & 42 & 21 \\
40 a 49 anos & 39 & 100 \\
50 a 59 anos & 30 &
\end{tabular}

Fonte: Silva LSR, et al., 2020. SINAN, 2019.

Embora a Hanseníase acometa ambos os sexos, a predominância do sexo masculino, como corroborado nos estudos de Santos DAS, et al. (2017) e Costa AKAN, et al. (2019), além de ter sido relatado no Boletim Epidemiológico do Ministério da Saúde (BRASIL, 2018), pode ser explicada devido ao maior contato social e ao trabalho, que contribuem para uma maior exposição ambiental e risco de contato com indivíduos doentes, favorecendo a elevação do número de casos de Hanseníase entre os homens (ROSA AM, et al., 2019). Além disso, a menor preocupação com a estética, relacionada à presença de lesões de pele provocadas pela doença, e o não comparecimento aos serviços de saúde ou em menor frequência do que as mulheres são fatores que dificultam o diagnóstico inicial e a realização do tratamento (GOIABEIRA YNLA, et al., 2018; QUARESMA MSM, et al., 2019).

A caracterização da doença por sexo permite indicar diferenças de acesso em termos da capacidade de alcance dos programas de saúde e da capacidade da população em utilizar os serviços de saúde. Além disso, é possível identificar variações na carga de Hanseníase entre os grupos populacionais e também discutir se estão ligadas a processos socioeconômicos, como a diferença de acesso e oportunidades (BRASIL, 2018).

No que se refere à faixa etária, mostra-se concordância com as pesquisas realizadas por Cunha DV, et al. (2019), Quaresma MSM, et al. (2019) e Rosa AM, et al. (2019). Conforme Basso MEM e Silva RLF (2017), o maior percentual de pessoas acometidas pela patologia estar na faixa etária de 30 a 39 anos indica que a 
população economicamente ativa é a mais afetada, o que caracteriza a Hanseníase como uma doença de adultos e adultos jovens. Observa-se, dessa forma, a importância da detecção precoce já que essa situação pode prejudicar a economia do município, visto que indivíduos incluídos nessa faixa etária podem vir a desenvolver incapacidades, lesões, estados reacionais, afastar-se da atividade produtiva e gerar um alto custo social (CUNHA DV, et al., 2019).

Em relação às características clínicas e epidemiológicas, a maioria absoluta dos pacientes notificados foi classificada como multibacilar com 123 casos, representando $85 \%$. Verificou-se que a forma clínica prevalente foi a Dimorfa (67\%), totalizando 97 casos. No que se refere ao modo de entrada, no período em estudo, foram notificados 104 casos novos de Hanseníase em adultos, o que correspondeu a $72 \%$ do modo de entrada no SINAN. Avaliando o modo de detecção, houve predominância da demanda espontânea com 60 casos (41\%). Quanto ao grau de incapacidade, a maioria dos casos $(n=60 ; 41 \%)$ foi notificada com grau de incapacidade zero. Já quanto ao tipo de saída, $62 \%$ dos pacientes $(n=90)$ obtiveram a cura (Tabela 3 ).

Tabela 3 - Caracterização clínico-epidemiológica dos casos de Hanseníase em pacientes adultos. 20162018.

\begin{tabular}{|c|c|c|}
\hline Variável & $\mathbf{n}$ & $\%$ \\
\hline \multicolumn{3}{|l|}{ Classificação operacional } \\
\hline Paucibacilar & 22 & 15 \\
\hline Multibacilar & 123 & 85 \\
\hline \multicolumn{3}{|l|}{ Forma Clínica } \\
\hline Indeterminada & 7 & 5 \\
\hline Tuberculoide & 15 & 10 \\
\hline Dimorfa & 97 & 67 \\
\hline Virchowiana & 22 & 15 \\
\hline Não classificada & 3 & 2 \\
\hline Ignorada & 1 & 1 \\
\hline \multicolumn{3}{|l|}{ Modo de entrada } \\
\hline Caso Novo & 104 & 72 \\
\hline Transferência do mesmo município & 7 & 5 \\
\hline Transferência de outro município (mesma UF) & 1 & 1 \\
\hline Transferência de outro estado & 1 & 1 \\
\hline Recidiva & 8 & 5 \\
\hline Outros ingressos & 24 & 16 \\
\hline \multicolumn{3}{|l|}{ Modo de detecção } \\
\hline Encaminhamento & 43 & 30 \\
\hline Demanda espontânea & 60 & 41 \\
\hline Exame contatos & 1 & 1 \\
\hline Outros modos & 1 & 1 \\
\hline Ignorado & 40 & 27 \\
\hline \multicolumn{3}{|l|}{ Grau de incapacidade } \\
\hline Grau 0 & 60 & 41 \\
\hline Grau 1 & 47 & 33 \\
\hline Grau 2 & 19 & 13 \\
\hline Não avaliado & 18 & 12 \\
\hline Branco & 1 & 1 \\
\hline \multicolumn{3}{|l|}{ Tipo de saída } \\
\hline Cura & 90 & 62 \\
\hline Transferência para mesmo Município & 7 & 5 \\
\hline Transferência para outro Município & 4 & 3 \\
\hline Óbito & 2 & 1 \\
\hline Abandono & 4 & 3 \\
\hline Erro Diagnóstico & 2 & 1 \\
\hline Ignorado & 36 & 25 \\
\hline Total & 145 & 100 \\
\hline
\end{tabular}

Fonte: Silva LSR, et al., 2020. SINAN, 2019. 
De acordo com o Ministério da Saúde (BRASIL, 2016), deve-se utilizar a classificação operacional de caso de Hanseníase visando definir o esquema de tratamento característico de cada tipo, denominado de poliquimioterapia (PQT), que se baseia no número de lesões cutâneas: os doentes são classificados em paucibacilares (PB) quando apresentam até cinco lesões de pele ou multibacilares (MB) quando há a presença de seis ou mais lesões de pele. Conforme a frequência apresentada, o fato de a maioria dos pacientes ter a forma MB indica um panorama epidemiológico de casos bacilíferos circulantes, os quais são considerados como principal fonte de transmissão da doença. As pessoas inseridas nessa classificação têm um quantitativo aumentado de bacilos na derme e em mucosas, e acabam liberando os bacilos no ambiente, o que por consequência leva a um maior número de portadores das formas graves da Hanseníase (SILVA MS, et al., 2014; VELÔSO DS, 2018).

No que diz respeito à forma clínica, o resultado encontrado assemelha-se com os estudos de Silva MS, et al. (2014), Goiabeira YNLA, et al. (2018) e Quaresma MSM, et al. (2019), onde se constatou a forma clínica Dimorfa como predominante. Segundo o Ministério da Saúde (BRASIL, 2017), a Dimorfa é a forma mais comum de apresentação da doença e potencialmente incapacitante, correspondendo a mais de $70 \%$ dos casos. Devido o seu longo período de incubação (cerca de 10 anos ou mais) e à lenta multiplicação do bacilo, acaba por acometer mais pessoas na idade adulta (BRASIL, 2017).

Esses dados revelam o diagnóstico tardio da doença, o qual contribui para a cadeia de transmissão com o surgimento de novos casos e para a manutenção da Hanseníase como um problema de saúde pública. Diante disso, é recomendada a detecção precoce dos casos a fim de iniciar o tratamento o mais breve possível, deste modo contribuindo para diminuir as complicações da doença e levando ao mínimo de alterações da qualidade de vida (VELÔSO DS, 2018). Detectar a doença nas formas clínicas multibacilares (dimorfa e virchowiana), consideradas as mais graves, leva ao risco de desenvolver incapacidades físicas perfeitamente evitáveis com o diagnóstico e tratamento nas fases iniciais da doença (BRASIL, 2017).

No que concerne ao modo de entrada, a notificação, em sua maioria, de casos novos indica que a cadeia de transmissão da doença está ativa no município. Considera-se caso novo de Hanseníase a pessoa que nunca recebeu qualquer tratamento específico para a doença e que apresenta os sinais e sintomas característicos desse agravo (BRASIL, 2016). Desse modo, a variável modo de entrada é bastante relevante na identificação de novos pacientes entre aqueles que convivem ou já conviveram com casos positivos.

Quanto ao modo de detecção dos casos novos, a maior parte dos casos notificados terem sido detectados por meio de demanda espontânea e um baixo percentual por meio de exame de contatos caracteriza a ocorrência de formas passivas de detecção. O exame de contatos é tido como um dos principais instrumentos de avaliação da Hanseníase, pois contribui de forma significativa para uma detecção mais precisa e precoce dos casos. Ele objetiva quebrar a cadeia de transmissão e evitar sequelas resultantes do diagnóstico tardio e da falta de acompanhamento adequado. Além disso, pretende descobrir possíveis fontes de infecção no domicílio ou fora dele, isso independentemente da classificação operacional do doente (BRASIL, 2017).

O fato de o próprio usuário procurar o serviço de saúde para obter o diagnóstico leva a inferir que a Atenção Primária à Saúde (APS) ainda apresenta dificuldades na detecção dos casos, e que as taxas de detecção poderiam ser elevadas caso fossem estabelecidas ações rotineiras de exame dos contatos dos pacientes. Esses dados indicam que a busca ativa de casos não está sendo implementada de forma satisfatória nos serviços de saúde, revelando uma falha na aplicação das diretrizes do plano de eliminação e tratamento da Hanseníase (GONÇALVES NV, et al., 2018).

No que diz respeito ao Grau de Incapacidade Física (GIF), assim como evidenciado nos estudos de Silva MS, et al. (2014), Santos DAS, et al. (2017) e Rosa AM, et al. (2019), o GIF predominante no momento do diagnóstico foi o grau zero. De acordo com o Ministério da Saúde (BRASIL, 2018), a classificação como incapacidade de grau zero ocorre quando a força muscular e a sensibilidade estão preservadas, sendo um indicador de que o diagnóstico, em sua maioria, está sendo precoce. No entanto, ao correlacionarmos a alta densidade de casos multibacilares encontradas neste estudo com a distribuição do GIF, apesar de o maior percentual de adultos portadores de Hanseníase apresentar Grau 0, a soma dos casos com Grau 1 e Grau 2 representa uma parcela significativa de pacientes com alguma incapacidade, o que confirma mais uma vez a detecção tardia da doença e sugere seu controle ineficaz. 
Sabe-se que as formas clínicas multibacilares constituem fatores de risco para as incapacidades físicas apresentadas pelos pacientes notificados, podendo provocar diminuição da força muscular ou diminuição de sensibilidade (Grau 1) e deformidade visível nas mãos, pés ou olhos (Grau 2) (BRASIL, 2018). A avaliação do GIF constitui uma importante ferramenta na identificação de pacientes com risco aumentado de reações e novas incapacidades. Dessa forma, é imprescindível que ele seja avaliado em todos os indivíduos acometidos pela doença no momento do diagnóstico e da cura (BRASIL, 2017). Também pôde ser constatado um número considerável de não avaliados, sendo esse um indicador que expõe falha na assistência dos profissionais de saúde ao negligenciarem a avaliação do GIF ou até mesmo despreparo para sua execução.

Já com relação ao tipo de saída, verificou-se que a maioria dos pacientes adultos (62\%) obteve a cura da doença. Segundo o Ministério da Saúde, é considerada uma pessoa de alta por cura o indivíduo que completa o esquema de tratamento de poliquimioterapia dentro dos prazos estabelecidos de acordo com a forma operacional da doença (BRASIL, 2016). Somado a esse percentual de cura, o baixo abandono do tratamento retrata a boa adesão à terapêutica proposta, a qual é fornecida de maneira gratuita pelos serviços de saúde pública. Esse resultado sugere a importância do acolhimento adequado do paciente que procura atendimento na unidade básica da APS, para que seja criado um vínculo entre ele e a equipe de saúde, orientando-o sobre a doença e a necessidade de concluir seu tratamento a fim de obter a cura (QUARESMA MSM, et al., 2019).

Os achados deste estudo evidenciaram que a presença, em sua maioria, das formas multibacilares e o baixo percentual de busca ativa dos portadores dessa patologia podem indicar manutenção da cadeia de transmissão no município maranhense, o que reforça a necessidade de integração das ações de controle principalmente na Atenção Primária, porta de entrada do Sistema Único de Saúde (SILVA MS, et al., 2014).

Conhecer os dados epidemiológicos de determinado local é de suma importância para que sejam desenvolvidas políticas públicas com foco na prevenção e controle da doença, identificando os grupos populacionais mais acometidos de maneira a iniciar o tratamento de forma precoce e prevenir incapacidades (VELÔSO DS, et al., 2018). Nesse contexto, é imprescindível implementar ações de vigilância epidemiológica, sendo necessário o aperfeiçoamento dos profissionais de saúde para identificação e acompanhamento dos casos por meio do reconhecimento dos sinais e sintomas da doença e realização de exame dos contatos, como também a correta notificação dos casos ao SINAN e orientação da população através de práticas de educação em saúde (PORTO MJ, et al., 2015; ARAÚJO RMS, et al., 2017).

\section{CONCLUSÃO}

A partir dos dados obtidos, pôde-se constatar que a Hanseníase acomete, em sua maioria, adultos do sexo masculino, na faixa etária de 30 a 39 anos, com classificação operacional multibacilar e forma clínica dimorfa, modo de entrada como caso novo, cuja detecção acontece em sua maioria por demanda espontânea, com grau de incapacidade zero e tipo de saída por cura. Esses resultados apontam para a necessidade da adoção de estratégias que contemplem o panorama da Hanseníase no município, além de investimentos no que diz respeito à capacitação e à atualização dos profissionais para a detecção precoce dos portadores da doença. Dessa forma, espera-se que este estudo forneça informações que permitam conhecer a dimensão da Hanseníase na coletividade, a fim de potencializar as medidas de prevenção e o pronto tratamento dos casos iniciais, o exame periódico dos contatos domiciliares do paciente e a educação em saúde para a população.

\section{REFERÊNCIAS}

1. ARAÚJO RMS, et al. Análise do perfil epidemiológico da hanseníase. Revista de Enfermagem, UFPE online. Recife, 2017; 11(9): 3632-41.

2. BASSO MEM, SILVA RLF. Perfil clínico-epidemiológico de pacientes acometidos pela hanseníase atendidos em uma unidade de referência. Revista da Sociedade Brasileira de Clínica Médica, 2017; 15(1): 27-32.

3. BRASIL. Ministério da Saúde. Secretaria de Vigilância em Saúde. Caracterização da situação epidemiológica da hanseníase em diferenças por sexo, Brasil, 2012-2016. Boletim Epidemiológico, 2018; 49(4): 1-10. 
4. BRASIL. Ministério da Saúde. Secretaria de Vigilância em Saúde. Departamento de Vigilância das Doenças Transmissíveis. Diretrizes para vigilância, atenção e eliminação da Hanseníase como problema de saúde pública: manual técnico-operacional. Brasília: Ministério da Saúde, 2016; 58 p.

5. BRASIL. Ministério da Saúde. Secretaria de Vigilância em Saúde. Departamento de Vigilância das Doenças Transmissíveis. Guia prático sobre a hanseníase. Brasília: Ministério da Saúde, 2017; 68 p.

6. COSTA AKAN, et al. Aspectos clínicos e epidemiológicos da hanseníase. Revista de Enfermagem UFPE on line, 2019; 13(1): 353-62.

7. CUNHA DV, et al. Perfil Epidemiológico da Hanseníase no Município de Castanhal - Pará no período de 2014 a 2017. Revista Eletrônica Acervo Saúde, 2019; 11(15): 1-8.

8. DATASUS. Departamento de Informática do Sistema Único de Saúde (SUS). Disponível em: http://www2.datasus.gov.br/DATASUS/index.php?area=02. Acesso em: 5 jun. 2019.

9. GOIABEIRA YNLA, et al. Perfil epidemiológico e clínico da Hanseníase em capital hiperendêmica. Revista de Enfermagem UFPE on line, 2018; 12(6): 1507-13.

10. GONÇALVES NV, et al. A hanseníase em um distrito administrativo de Belém, estado do Pará, Brasil: relações entre território, socioeconômica e política pública em saúde, 2007-2013. Revista Pan-Amaz Saúde, 2018; 9(2): 21-30.

11. LAFAIETE C. Hanseníase: Brasil permanece em $2^{2}$ posição no registro de novos casos. In: Portal PEBMED, 2019. Disponível em: https://pebmed.com.br/hanseniase-brasil-permanece-em-2a-posicao-no-registro-de-novos-casos/. Acesso em: 2 set. 2019.

12. PASSOS CEC, et al. Hanseníase no estado do Maranhão: análise das estratégias de controle e os impactos nos indicadores epidemiológicos. Revista Brasileira de Geografia Médica e da Saúde, Hygeia, 2016; 12(22): 88-100.

13. PORTO MJ, et al. Análise dos números de casos notificados de hanseníase pelo SINAN na Bahia. Revista Enfermagem Contemporânea, 2015; 4(2):137-144.

14. QUARESMA MSM, et al. Perfil clínico e epidemiológico dos pacientes portadores de hanseníase em uma unidade de referência no estado do Pará. Revista Eletrônica Acervo Saúde, 2019; 18(18): 1-10.

15. RIBEIRO MDA, et al. Estudo epidemiológico da hanseníase no Brasil: reflexão sobre as metas de eliminação. Revista Panamericana de Salud Publica, 2018; 42(7): 1-7.

16. ROSA AM, et al. Perfil clínico-epidemiológico da hanseníase no interior maranhense no período de 2013 a 2017. Prevenção e promoção de saúde, v. 3. Ponta Grossa: Atena Editora, 2019; 28 p.

17. SANTOS DAS, et al. Prevalência de casos de hanseníase. Revista de Enfermagem UFPE on line, 2017; 11(Supl.10): 4045-55.

18. SILVA MS, et al. Perfil clínico-epidemiológico da hanseníase no estado do Acre: estudo retrospectivo. Revista Hansenologia Internationalis, 2014; 39(2): 19-26.

19. SOUZA EA, et al. Hanseníase e gênero no Brasil: tendências em área endêmica da região Nordeste, $2001-2014$. Revista de Saúde Pública, 2018; 52(20): 1-12.

20. VELÔSO DS. Perfil clínico-epidemiológico da hanseníase no estado do Piauí, no período de 2009 a 2016. Dissertação (Mestrado em Medicina Tropical) - Instituto Oswaldo Cruz, Teresina, 2018; 126 p.

21. VELÔSO DS, et al. Perfil Clínico Epidemiológico da Hanseníase: Uma Revisão Integrativa. Revista Eletrônica Acervo Saúde, 2018; 10(1): 1429-1437. 